\title{
$\underline{\text { Safety and effectiveness of long-acting injectable Aripiprazole in daily practice: a naturalistic }}$
} $\underline{\text { retrospective study }}$

Samr Dawood $^{\mathrm{a}^{*}}$, Jared G. Smith ${ }^{\mathrm{b}}$, Nighat Nadeem ${ }^{\mathrm{c}}$, Cyrus Abbasian ${ }^{\mathrm{c}}$

${ }^{a}$ South London and The Maudsley NHS Trust, Lewisham Hospital ,_Lewisham High Street, London, SE13 6LH, UK

${ }^{\mathrm{b}}$ Population Health Research Institute, St George's, University of London, Cranmer Terrace, London SW17 ORE, UK

${ }^{c}$ South West London and St George's Mental Health NHS Trust, 61 Glenburnie Road, London, SW17 7DJ, UK

Short running title: Safety and effectiveness of Aripiprazole Depot

*Corresponding author (please address proofs and reprints to):

Dr Samr Dawood

South London and The Maudsley NHS Trust

Lewisham Hospital

Lewisham High Street

London, SE13 6LH

E-mail address: samr.dawood@slam.nhs.uk 


\section{Funding source}

This research did not receive any grant from funders in the public, commercial, or not-for-profit sectors.

\section{Conflicts of interest}

None of the authors have any conflicts of interests to disclose.

\section{Acknowledgments}

We would like to thank Sophie Quarshie, Sophie Bamber, and Nehal Patel for their assistance with data collection. 


\begin{abstract}
:
Background and objectives: It was postulated that Aripiprazole may cause impulse-control problems. Therefore, real-world data on the effects and side effects of Aripiprazole 400mg depot are needed to review the utility, safety and the clinical effectiveness of Aripriprazole 400mg depot and highlight the occurrence of adverse effects including impulsivity in a naturalistic treatment settings.
\end{abstract}

Methods: A non-interventional, retrospective study was conducted to examine the electronic records of all patients who were receiving or had received Aripiprazole Lauroxil in South West London before May 2017. Disorder symptoms and side effects were compared between pre- and post-Aripiprazole PO and Aripiprazole Depot treatments.

Results: Data were considered for 57 patients who switched to Aripiprazole depot due to non-compliance and/or side effects. Most were suffering from chronic schizophrenia (75.4\%) and half (50.9\%) had comorbid alcohol and/or illicit drug use. Oral Aripiprazole was effective in improving hallucinations, delusions and depressive symptoms $(P<0.022)$; these benefits were maintained or enhanced further with Aripiprazole Depot. Proportions of patients experiencing sexual disinhibition, aggression and deliberate self-ham decreased significantly $(P<0.003)$ after starting oral Aripiprazole with no further change after Aripiprazole Depot. By study end, a quarter of patients had stopped taking Aripiprazole Depot due to ineffectiveness, side effects or patient request.

Conclusion: Aripriprazole Lauroxil 400mg depot was safe, effective and well tolerated. Side effects manifestations should prompt full evaluations and dose reduction should be considered.

\title{
$\underline{\text { Keywords }}$
}

Antipsychotic, Aripiprazole, depot, long-acting, effects, impulse control 


\section{Introduction}

Aripiprazole was introduced more than 12 years ago and has a complex mechanism of action; through agonism, partial agonsim and antagonism at dopamine and serotonin receptors ${ }^{1,2}$. Its principal mechanism of action is partial agonism at the D2 dopamine receptor at which it has high affinity ${ }^{3}$. This partial agonism can reduce D2 hyperactivity in the mesolimbic pathway and alleviate positive symptoms of schizophrenia whilst still providing enough D2 receptor stimulation in the mesocortical and nigrostriatal pathway to prevent both extrapyramidal side effects and negative symptoms ${ }^{4}$. Research suggests that patients with negative symptoms of psychosis may respond better to Aripiprazole than Risperidone ${ }^{5}$ and depot injection of Aripiprazole could help prevent clinical deterioration, improves treatment adherence and decreases relapses in patients with poor compliance ${ }^{6}$.

However, during the last few years, dopamine replacement therapy (DRT) have become a wellknown risk factor for developing an impulse control disorder ${ }^{7}$. Aripiprazole might resemble dopaminergic medications as it has partial agonist action at D2 receptors ${ }^{1,2}$, but little is known about the associations between Aripiprazole and impulse control disorders. However, published case reports suggest a link ${ }^{7}$. Although, impulse control disorders as adverse effects of Aripiprazole are rare, they are potentially harmful to patients if they are not recognised. The United States FDA has issued a warning that the use of Aripiprazole may be associated with compulsive behaviours or uncontrollable urges such as compulsive eating, shopping, gambling and sexual actions ${ }^{8}$. These can affect any patient taking Aripiprazole and are reported to stop when Aripiprazole is stopped or the dose is decreased. The FDA warning is based on an analysis of data available through the FDA Adverse Event Reporting System (FAERS). This data suggests that since the approval of the first Aripiprazole product (Abilify) in November 2002, 184 case reports have been identified in which there was an association between the use of Aripiprazole and impulse-control problems. Of these cases, pathological gambling was the most common and contributed to 164 of these cases. As the FAERS includes reports that are only submitted to the FDA, there may be many more unreported cases ${ }^{8}$. These concerns were supported by a recent review which described 22 cases of impulse control problems associated with the initiation of treatment with Aripiprazole and the majority of patients had pathological gambling followed by hyper-sexuality ${ }^{9}$. The review noted that those adverse effects of Aripiprazole were associated with higher doses ${ }^{9}$. On the other hand, there are reports in the literature showing that the use of Aripiprazole can decrease drinking behaviour in alcohol-dependent patients and research suggests that Aripiprazole could play a role in the treatment of alcohol dependence ${ }^{6}$. This is supported by studies which suggest that partial dopamine D2/D3 receptor agonists reduce the compulsive urges and impulsivity which is characteristic of addiction behaviours ${ }^{3,5}$. Also, impulse control disorders are common in psychiatric patients, and one-third of adult psychiatric inpatients might be suffering from a comorbid impulse control disorder ${ }^{10}$. However, only $1.5 \%$ of them have a formal diagnosis of impulse control 
disorder on admission which suggests that these disorders often remain unrecognised ${ }^{10}$. Impulse control disorders are reported to be associated with more severe psychiatric illnesses as well as being more common in young adults ${ }^{11}$ and in females ${ }^{12}$.

Aims of the study

Ultimately, case reports do not reflect the true prevalence of these side effects. Therefore, additional research is needed collating basic epidemiological data of the occurrence of side effects in patients receiving Aripiprazole whilst not manipulating any variables or testing hypotheses. The aims of the present study were to review the utility, safety and the clinical effectiveness of Aripiprazole Lauroxil depot and to highlight the occurrence of the adverse effects, including the problem of impulsivity.

\section{Material and Methods}

Sample

A non-interventional, retrospective, observational study was conducted to determine the safety and effectiveness of Aripiprazole Lauroxil $400 \mathrm{mg}$ once-monthly extended-release injection in every day clinical practice. This project investigated all patients under the care of the NHS trust which provides mental health services to south west London who were receiving or had received Aripiprazole Lauroxil in or before May 2017. Aripiprazole depot injections are not available through General Practitioner services and are dispensed by the mental health trust's pharmacy department for patients who have been prescribed it by the mental health trust's Psychiatrists. As the Pharmacy department maintains a record of all patients on Aripiprazole Depot, a list of 64 patients was obtained from the Pharmacy department. This was considered to be the sample population.

Procedure.

The medical notes of all Trust patients receiving Aripiprazole depot were examined by clinical team members as part of routine clinical practice to evaluate the safety and effectiveness of the drug. After extraction of relevant information, data was pseudo-anonymised ensuring patient confidentiality and aggregated for the purposes of analyses. This study would not fall under 'research' because it used routinely collected clinical data (medical notes) accessed only by members of the clinical team and was retrospective in design - as such, the National REC would not have considered this study for ethical approval and was registered as a service evaluation with the local Trust's Clinical Effectiveness Team and its results were presented during the local academic program runs by the trust.

To extract data efficiently from the case records to answer the clinical query we need to agree on the variables to be extracted. As the electronic clinical records include enormous entries clinical and non-clinical by different professionals and to ensure complete and all-encompassing browsing for the relevant texts, the search of the electronic 
Safety and effectiveness of Aripiprazole Depot clinical records involved two stages. Firstly, key-words were used to locate the relevant clinical entries using a search instrument in the electronic record system, which would highlight the key wards in all the records. The following search terms were used: medication, Aripiprazole, depot, maintenance, antipsychotic, hallucination, delusion, psychosis, low mood, sadness, depression, elation, negative symptoms, cognitive, Parkinson's, tremor, rigidity, akathesia, restlessness, tardive dyskinesia, suicide, self-harm, gambling, impulse control, sexual, dis-inhibition and aggression. Once the relevant entries had been highlighted, these were manually screened to extract the relevant information. Most patients in our sample were on oral Aripiprazole before starting Aripiprazole depot as that was a requisite by the pharmacy to stabilize their conditions before switching to Aripiprazole IM depot formula.

Clinical parameters

Clinical parameters to determine the range of the positive effects on symptomatology were gathered before and after the initiation of both oral and Aripiprazole long-acting injection (L Data related to the effects and the adverse effects of the therapy were collected eight weeks after the commencement of the maximum doses of oral Aripiprazole, and LAD Aripiprazole. Patient sociodemographic data and basic clinical details including diagnoses, comorbidities, psychiatric hospitalisations, concomitant medication use and substance abuse/dependency were also extracted. Reasons for initiation and discontinuation of Aripiprazole therapy were also noted.

Statistical analysis

The frequencies (percentages) of individuals with disorder symptoms and side effects were calculated at pre/post- Aripiprazole PO and Aripiprazole Depot treatments and the McNemar mid- $P$ test for binary matchedpair data ${ }^{13}$ was used to compare proportions. Comparisons between groups concerning outcome of Aripiprazole Depot treatment (stopped taking versus ongoing, presence of (any) side effect following treatment versus no side effects) were made using chi-squared tests, Fisher's exact tests or independent group t-tests according to data distribution. Where skewness and kurtosis estimates indicated that continuous variables did not approximate a Gaussian distribution ${ }^{14}$, Mann-Whitney $U$ tests were employed for group comparisons. To control for the possibility of false-positives because of multiple pre- to post-treatment testing across disorder symptoms and side effects, the false discovery rate (FDR; ${ }^{15}$ ) approach was applied to within-group comparisons, with control set to 5\% (i.e., a ranked order of ascending $P$ values obtained from $t$-tests were compared to a series of threshold values of significance obtained from an FDR analysis). For all 
other analyses, a criterion for statistical significance was set at $P<0.05$. All statistical analyses were performed using SPSS (IBM; Version 24).

\section{Results}

Data from electronic medical records for 64 patients under the care of South West London and St George's Mental Health Trust who were registered as receiving Aripiprazole Lauroxil (Aripiprazole Depot) were extracted. Seven patients' notes revealed they had not received Aripiprazole Depot - as such, data was considered for 57 patients only.

The sociodemographic characteristics and clinical data of participants are shown in Table 1. A small majority of patients were male, with a wide age range. More than three-quarters of the patients were unemployed, although most lived in independent accommodation. Schizophrenia was the most common diagnosis in the patient sample, with schizoaffective, delusional and bipolar disorder diagnoses much less frequent. Substance abuse disorder was diagnosed in 5 patients, although this was a comorbid with schizophrenia or bipolar disorder in 3 of these cases. A little less than half of patients smoked while $50.9 \%$ of patients reported (illicit) drug use and/or alcohol abuse. A small number of patients were obese or suffered from hypertension. Prior to administration of Aripiprazole (PO), patients were taking a wide variety of (antipsychotic) medications, although Olanzapine and Risperidone were most commonly administered.

\section{Insert Table 1 about here}

Aripiprazole PO and Depot Treatment

All but 3 (54/57) patients' data indicated they had received Aripiprazole PO prior to Depot (one patient refused PO, another went immediately to Aripiprazole Depot from Olanzapine OD, and one patient who began Aripiprazole Depot at another Trust did not have data available concerning administration of Aripiprazole PO); duration of administration varied widely, although was less than a year for most patients, and more than $80 \%$ of patients were on a maximum dosage of $15 \mathrm{mg}$ or less. Patients switched from Aripiprazole PO to Depot predominantly because of side effects and/or compliance issues (Table 2), with 51 of 54 patients receiving Depot within 10 weeks after ending administration of PO.

Insert Table 2 about here 
Safety and effectiveness of Aripiprazole Depot Just over a quarter of patients had stopped Aripiprazole Depot prior to the study end period due to ineffectiveness, side effects (akathisia (2), tremor (1) and stiffness (1)), non-compliance or patient request. Of the 54 patients administered Aripiprazole PO, 11 stopped PO before 8 weeks while one patient had no symptom data available at Post-Aripiprazole PO; as such, data at Post-Aripiprazole was considered for 42 patients only. Pre-Aripiprazole Depot data was considered for 56 patients in the 8-week period prior to first Depot administration; this included the 11 patients who stopped PO before 8 weeks and a small number of patients (8) who had more than a month between stopping PO and administration of Depot. Those patients stopping Aripiprazole Depot had taken Aripiprazole PO prior to Depot for a shorter time than those continuing (Median 1.8 months (range 0.62-76.0) versus 12.8 months (range 0.4-117.0); $\mathrm{P}=$ $0.003)$ and were less likely to be unemployed $(53.3 \%$ versus $85.7 \% ; \mathrm{P}=0.010)$. There were no other sociodemographic or clinical factors related to stopping Aripiprazole Depot.

Symptoms and Side Effects of Aripiprazole treatment

The frequency (percentage) of patients presenting with symptoms prior to and in the 8 week period after administration of Aripiprazole PO and Depot treatment are shown in Table 3. From pre- to post-Aripiprazole PO, the prevalence of hallucinations, delusions, and depression all approximately halved. Further significant decreases from pre- to post-Aripiprazole Depot were found for hallucinations and depression. Across both Aripiprazole PO and Depot treatments, decreases in presentations of elation and negative symptoms were found, although (pre-treatment) floor effects precluded significant differences.

Insert Table 3 about here

The frequency (percentage) of patients experiencing side effects prior to receiving Aripiprazole and in the 8-week period following administration of Aripiprazole PO and Depot treatment are shown in Table 4. There were highly significant decreases in aggression, sexual disinhibition and deliberate selfharm/suicide attempts post Aripiprazole PO administration, which were largely maintained post Aripiprazole Depot. In the 8 weeks following both Aripiprazole PO and Depot treatments, presentations of Parkinsonism, tardive dyskinesia and gambling were infrequent, with no significant differences compared with pre-Aripiprazole. Impulsive behaviour (not assessed at before Aripiprazole treatment) affected less than one in 10 patients in the 8 weeks following administration of Aripiprazole PO and Aripiprazole Depot. Notably, presence of any side effect post Aripiprazole Depot was not associated with any sociodemographic or (baseline) clinical variable, including diagnosis, alcohol abuse and illicit drug use, although patients 

experiencing side effects after receiving Aripiprazole Depot were more likely than those with no side effects to have experienced side effects post Aripiprazole PO (38.1\% versus $13.9 \%$; $P=0.036)$.

Insert Table 4 about here

\section{Discussion}

Our sample represents patients who were considered by their clinicians to be in need of Aripiprazole LAI, which suggests that non-adherence in the context of other factors necessitated the choice of initiating this treatment. Non-adherence with antipsychotic medications is an important avoidable factor that can lead to deteriorations in mental state and antipsychotic medications with long acting injection (LAI) formula were developed to reduce the problem of non-adherence. ${ }^{16}$ Our data showed that in our sample the factors leading to a switch to Aripiprazole were non-compliance and/or side effects, which matches previous findings. ${ }^{17}$ Also, a systematic review concluded that tolerability and non-efficiency was the main reason for a switch to Aripiprazole from other antipsychotics. ${ }^{18}$ The switch to Aripiprazole in our cohort followed use of different antipsychotics across the sample but mostly Olanzapine, Risperidone, Paliperidone, and Zuclopenthixol. High percentages of physical health problems in our sample were noticeable and it might be a factor in choosing Aripiprazole Depot to avoid metabolic side effects. ${ }^{4}$ Schizophrenia was the main diagnostic category. It was reported previously that poor adherence was associated with a diagnosis of schizophrenia. ${ }^{19}$ surprisingly Bipolar disorder was represented by a small percentage despite the increasing amount of literature recommending the usage of Aripiprazole, including Aripiprazole LAI, in Bipolar disorder. ${ }^{20}$

Interestingly, our data showed significant improvement in patients' delusions and hallucinations after the commencement of Aripiprazole therapies. However our results are reliant on data extracted retrospectively from clinical notes of a small number of patients, while a comprehensive systemic review of randomised controlled trials concluded Aripiprazole is less effective, at least in comparison with Olanzapine. ${ }^{18}$ Also, oral Aripiprazole appeared effective in treating low mood and elation. Previous studies suggested that Aripiprazole is useful in treating depression, especially in low doses. ${ }^{21}$ Three out of nine patients in our sample showed improvement in negative symptoms with oral Aripiprazole and two out of four patients showed improvement in negative symptoms with the LAI. The most hoped-for effect of Aripiprazole on negative symptoms is probably dose related. ${ }^{22}$

Our retrospective clinical notes review study was designed primarily to answer the question about the frequency of impulse control problems associated with the use of Aripiprazole LAI in everyday practice. The control of impulsive behaviour is a multidimensional concept which can be subdivided into separate problems. We considered the following clinical symptoms as parameters to gauge these problems; gambling, 
Safety and effectiveness of Aripiprazole Depot sexual disinhibition, and aggression because these warning signs are related to the most important impulsivity reported with the use of the antipsychotic drug Aripiprazole (Abilify, Abilify Maintena, Aristada, and generics). ${ }^{6}$ We excluded binge eating and excessive shopping from the list of the parameters to avoid the underreporting bias. In our sample, only one patient had a gambling problem before starting Aripiprazole treatment. This disappeared after commencing oral Aripiprazole; however it re-emerged again after starting Aripiprazole LAI. High rates of sexual disinhibition (28.1\%), and aggression (57.9\%) were recorded before initiating oral Aripiprazole, but they were reduced significantly with oral Aripiprazole treatment. It is important to take in consideration dosage as around $80 \%$ of patients received $15 \mathrm{mg}$ or lower maximum doses of Aripiprazole. However, a small numerical increase in the rates of sexual dis-inhibition (3.5\%) and aggression (7\%) was noted with the use of Aripiprazole LAI. A change in the rate of deliberate self-harm/suicidality, which went down from $21.1 \%$ to $3.7 \%$ after the initiation of oral Aripiprazole, was also observed. These observations are broadly comparable with a recent finding that impulsive control disorders associated with higher oral doses of Aripiprazole ${ }^{6,23}$ and appeared to be the strongest for the depot formulation of Aripiprazole. ${ }^{24}$ Low doses of Aripiprazole have been successfully used in the treatment of trichotillomania $^{25}$ and a meta-analysis has concluded that Aripiprazole has a much larger effect-size than other antipsychotics in controlling impulsivity and anger related symptoms. ${ }^{26}$ It should be noted that because of the retrospective design of this study, the impulse control problems detailed in the results were overt symptoms recognized and recorded by healthcare professionals, and patients had not been formally diagnosed with an impulse control disorder. It is possible that healthcare professionals were not fully aware of the importance of clarifying these impulsive control problems or the symptoms were below the threshold of formal diagnoses. In addition, the much higher rates of all types of impulse control problems prior to Aripiprazole initiation instigates a dilemma of the possible origin of these impulsivity symptoms, as they may be part of the nature of the psychiatric illnesses or part of the patient's premorbid personality traits. ${ }^{27}$

Other indicators for safety and tolerability used in this study were extrapyramidal symptoms and we focused separately on tardive dyskinesia (TD) because of the importance of this side effect. The clinical records indicated that the total percentage of people who had TD in our sample before switching to Aripiprazole was $8.8 \%$, which is higher than the average. ${ }^{28}$ However, our results showed that four out of five patients who had TD showed improvement in their symptoms after starting the stabilizing face of oral Aripiprazole and this improvement continued with the depot formulation. Recordings the presence or the improvement of (TD) were based on the clinical impression of the medical professionals. No rating scales were used. Previous reports had mixed results but suggest that Aripiprazole may be used as a treatment for TD. ${ }^{29}$ The total percentage of patients with extrapyramidal symptoms in our sample before the initiation of the Aripiprazole therapy (22.8\%) was very close to the percentage of extrapyramidal symptoms in patients receiving Aripiprazole $400 \mathrm{mg}$ depot recorded in a previous study.$^{30} \mathrm{In}$ our sample, however, the total percentage of patients with extrapyramidal symptoms dropped to $14.9 \%$; this was mostly because of the improvement in dyskinesia. Interestingly, a randomised controlled trial found that $21.9 \%$ of patients in an Aripiprazole once- 

monthly $400 \mathrm{mg}$ group, $11.7 \%$ of patients in an oral Aripiprazole group and 12.2\% of patients in an Aripiprazole once-monthly $50 \mathrm{mg}$ group had treatment-emergent extrapyramidal symptoms which were mostly related to Akathisia. ${ }^{30}$ Nevertheless that study used rating scales to detect Parkinsonian features while our results were extracted retrospectively from everyday clinical notes whereby detection of those extrapyramidal symptoms was dependent on patient reporting and clinicians' subjective judgments.

Lastly, Aripiprazole LAI is not appropriate for patients who have not tolerated the oral form either currently or in the past and the manufacturer recommended to establish tolerability with 2 weeks oral aripiprazole prior to initiating a depot formula; however the 2 -week interval may be insufficient. It is possible extended trials of oral Aripiprazole therapy could decrease the chance of non-adherence with the depot formula, as our study showed that those patients who stopped Aripiprazole Depot had taken Aripiprazole PO prior to Depot for a shorter time than those continuing. This might be because side effects continued to emerge weeks after the start of the treatment, ${ }^{31-33}$ and as indicated by the present findings, patients experiencing side effects after receiving oral Aripiprazole are more likely to suffer from side effects with Aripiprazole Depot.

\section{Strengths and Limitations}

The major strength of this study is that it included all patients receiving Aripiprazole in South West London, which limits the possibility of selection bias and expectation bias. Also, as the patients' clinical notes representing real world events were thoroughly reviewed, there was very limited attribution bias, although it is difficult to compare our results with other studies. ${ }^{34}$ The main limitations of this study relate to its retrospective design. This was a naturalistic, non-randomized study that did not include placebo or (active) control groups, which limits the ability to determine whether positive and/or adverse effects were specifically related to (Aripiprazole) medication or a consequence of other (unforeseen) factors such as the influence of concomitant psychoactive medications, or regression to the mean. Further, as data were already entered by healthcare staff without a methodology to ensure standardization and accurate measurement at the time of data entry, data quality control was limited. However, it is worth considering that, in general, naturalistic studies yield findings similar to randomised control studies, ${ }^{35}$ and although data resources including electronic patient records are not shaped by research priorities, ${ }^{36}$ the positive predictive value of electronic patient records has previously been reported as high. ${ }^{34}$ In this study, consideration of symptomatic changes and presence of adverse effects was limited to the 8-week period following administration of Aripiprazole PO and Depot treatment, and it is possible that for some patients, problematic symptoms and/or side effects emerged after this period. Finally, the dose of the oral Aripirazole varied widely across the sample and for some patients during the study period.

\section{Conclusions}


Safety and effectiveness of Aripiprazole Depot This study showed that in practice Aripiprazole prolonged-release suspension for injection can be a safe treatment option. Side effects were not prevalent and mostly were not serious. However, mental health specialists should monitor side effects and patients or their caregivers must specifically be educated about the possibility of side effects.

\section{$\underline{\text { Significant Outcomes }}$}

1. The main factors leading to a switch to Aripiprazole from other antipsychotics were non-compliance, side effects and limited effectiveness.

2. There was a significant improvement in delusions and hallucination with Aripiprazole treatment. Symptoms of tardive dyskinesia also improved with Aripiprazole in 4 out of 5 patients.

3. Rates of sexual disinhibition, aggression, deliberate self-harm (DSH)/suicidal attempt were significantly reduced with oral Aripiprazole and did not significantly increase in the rates of these with the use of Aripiprazole depot.

\section{$\underline{\text { Limitations }}$}

The main limitation is data quality assurance as data were extracted from patients' clinical notes which was already entered by healthcare staff without a methodology to ensure standardization and accurate measurement at the time of data entry.

\section{$\underline{\text { Ethical statement }}$}

This paper collected data from patient's clinical notes, and this was done internally as part of an audit/evaluation, so as to improve our quality of care. Those patients were receiving routine care and had been treated already according to the national and the local guidelines and the paper does not report on the use of experimental or new protocols. Hence we did not seek/obtain ethical approval for a study but rather project approval from the local mental health trust authority.

The efficacy and side effects of Aripiprazole have been demonstrated in a number of randomised, controlled trials, however, due to strict inclusion and exclusion criteria, these studies may not be representative of day to day clinical practice. Also, in particular the recent reports about the association between Aripiprazole and impulsivity have not been studies beyond the events of case reports. Accordingly this paper will have an important addition to the current states of the clinical knowledge, and it might instigate further investigations.

Because of the nature of this work, It is has biases that are associated with retrospective studies however, it still has value and can create a structure for a prospective study, if appropriate. 


\section{Funding source}

Safety and effectiveness of Aripiprazole Depot

This research did not receive any grant from funders in the public, commercial, or not-for-profit sectors.

\section{Conflicts of interest}

None of the authors have any conflicts of interests to disclose.

\section{References}

1. Brust TF, Hayes MP, Roman DL, Watts VJ. New functional activity of aripiprazole revealed: Robust antagonism of D2 dopamine receptor-stimulated G $\beta \gamma$ signaling. Biochemical pharmacology. 2015;93(1):85-91.

2. de Bartolomeis A, Tomasetti C, Iasevoli F. Update on the mechanism of action of aripiprazole: translational insights into antipsychotic strategies beyond dopamine receptor antagonism. CNS drugs. 2015;29(9):773-799.

3. Vergne DE, Anton RF. Aripiprazole: a drug with a novel mechanism of action and possible efficacy for alcohol dependence. CNS \& Neurological Disorders-Drug Targets (Formerly Current Drug Targets-CNS \& Neurological Disorders). 2010;9(1):50-54.

4. Taylor DM, Barnes TR, Young AH. The Maudsley prescribing guidelines in psychiatry. John Wiley \& Sons; 2018.

5. Farnia V, Shakeri J, Tatari F, et al. Randomized controlled trial of aripiprazole versus risperidone for the treatment of amphetamine-induced psychosis. The American journal of drug and alcohol abuse. 2014;40(1):10-15.

6. Peters-Strickland T, Baker RA, McQuade RD, et al. Aripiprazole once-monthly $400 \mathrm{mg}$ for longterm maintenance treatment of schizophrenia: a 52-week open-label study. npj Schizophrenia. 2015;1:15039.

7. Grall-Bronnec M, Sauvaget A, Perrouin F, et al. Pathological gambling associated with aripiprazole or dopamine replacement therapy: do patients share the same features? A review. Journal of clinical psychopharmacology. 2016;36(1):63. 
Safety and effectiveness of Aripiprazole Depot

8. Food U, Administration D. FDA drug safety communication: FDA warns about new impulse-control problems associated with mental health drug aripiprazole (Abilify, Abilify Maintena, Aristada). 2016 May 3. From FDA website.

9. Mahapatra A, Sharma P, Sagar R. Aripiprazole induced Impulse Control Disorders: Where do we stand? Asian journal of psychiatry. 2016;23:128-130.

10. Grant JE, Levine L, Kim D, Potenza MN. Impulse control disorders in adult psychiatric inpatients. American Journal of Psychiatry. 2005;162(11):2184-2188.

11. Odlaug BL, Grant JE. Impulse-control disorders in a college sample: results from the selfadministered Minnesota Impulse Disorders Interview (MIDI). Primary care companion to the Journal of clinical psychiatry. 2010;12(2).

12. Grant JE, Williams KA, Potenza MN. Impulse-control disorders in adolescent psychiatric inpatients: co-occurring disorders and sex differences. The Journal of clinical psychiatry. 2007.

13. Fagerland MW, Lydersen S, Laake P. The McNemar test for binary matched-pairs data: mid-p and asymptotic are better than exact conditional. BMC medical research methodology. 2013;13(1):91.

14. Hair JF, Anderson RE, Tatham RL, Black WC. Multivariate data analysis. Upper Saddle River1998.

15. Benjamini Y, Hochberg Y. Controlling the false discovery rate: a practical and powerful approach to multiple testing. Journal of the Royal Statistical Society Series B (Methodological). 1995:289-300.

16. Bellack AS, Bowden CL, Bowie CR, et al. The expert consensus guideline series: adherence problems in patients with serious and persistent mental illness. Journal of Clinical Psychiatry. 2009;70(SUPPL. 4):1-48.

17. Kane JM. Treatment of schizophrenia. Schizophrenia Bulletin. 1987;13(1):133-156.

18. Komossa K, Rummel-Kluge C, Schmid F, et al. Aripiprazole versus other atypical antipsychotics for schizophrenia. Cochrane Database of Systematic Reviews. 2009(4).

19. Baylé FJ, Tessier A, Bouju S, Misdrahi D. Medication adherence in patients with psychotic disorders: an observational survey involving patients before they switch to long-acting injectable risperidone. Patient preference and adherence. 2015;9:1333.

20. Torres-Llenza V, Lakshmin P, Lieberman DZ. Spotlight on once-monthly long-acting injectable aripiprazole and its potential as maintenance treatment for bipolar i disorder in adult patients. Neuropsychiatric disease and treatment. 2018;14:285.

21. Han C, Wang S-M, Kwak K-P, et al. Aripiprazole augmentation versus antidepressant switching for patients with major depressive disorder: a 6-week, randomized, rater-blinded, prospective study. Journal of psychiatric research. 2015;66:84-94.

22. James S, Kapugama C, Al-Uzri M. Use of aripiprazole long acting injection in negative symptoms of schizophrenia. Case reports in psychiatry. 2016;2016. 
Safety and effectiveness of Aripiprazole Depot

23. Feltenstein MW, Altar CA, See RE. Aripiprazole blocks reinstatement of cocaine seeking in an animal model of relapse. Biological psychiatry. 2007;61(5):582-590.

24. Lertxundi U, Hernandez R, Medrano J, Domingo-Echaburu S, Garcia M, Aguirre C. Aripiprazole and impulse control disorders: higher risk with the intramuscular depot formulation? International clinical psychopharmacology. 2018;33(1):56-58.

25. Sasaki T, Iyo M. Treatment of puberty trichotillomania with low-dose aripiprazole. Annals of general psychiatry. 2015;14(1):18.

26. Mercer D, Douglass AB, Links PS. Meta-analyses of mood stabilizers, antidepressants and antipsychotics in the treatment of borderline personality disorder: effectiveness for depression and anger symptoms. Journal of personality disorders. 2009;23(2):156-174.

27. Kisa C, Yildirim SG, Göka E. Impulsivity and mental disorders. Turk psikiyatri dergisi= Turkish journal of psychiatry. 2005;16(1):46-54.

28. Correll CU, Schenk EM. Tardive dyskinesia and new antipsychotics. Current opinion in psychiatry. 2008;21(2):151-156.

29. Kang N-R, Kim M-D. Tardive dyskinesia: treatment with aripiprazole. Clinical Psychopharmacology and Neuroscience. 2011;9(1):1.

30. Fleischhacker WW, Sanchez R, Perry PP, et al. Aripiprazole once-monthly for treatment of schizophrenia: double-blind, randomised, non-inferiority study. The British Journal of Psychiatry. 2014;205(2):135-144.

31. Hawley C, Turner M, Latif MA, Curtis V, Saleem PT, Wilton K. Switching stable patients with schizophrenia from depot and oral antipsychotics to long-acting injectable risperidone: reasons for switching and safety. Human Psychopharmacology: Clinical and Experimental. 2010;25(1):37-46.

32. Matsumoto H, Ishigooka J, Ono H, Tadori Y. Safety and efficacy from a 6-week double-blind study and a 52-week open-label extension of aripiprazole in adolescents with schizophrenia in Japan. Psychiatry and clinical neurosciences. 2018;72(9):701-712.

33. Wang S-M, Han C, Lee S-J, Patkar AA, Masand PS, Pae C-U. Schizophrenia relapse and the clinical usefulness of once-monthly aripiprazole depot injection. Neuropsychiatric disease and treatment. 2014;10:1605.

34. Thiru K, Hassey A, Sullivan F. Systematic review of scope and quality of electronic patient record data in primary care. $B M J .2003 ; 326(7398): 1070$.

35. Golder S, Loke YK, Bland M. Meta-analyses of adverse effects data derived from randomised controlled trials as compared to observational studies: methodological overview. PLoS medicine. 2011;8(5):e1001026.

36. Stewart R, Davis K. 'Big data' in mental health research: current status and emerging possibilities. Social psychiatry and psychiatric epidemiology. 2016;51(8):1055-1072. 
Table 1. Socio-demographic data and clinical profile for participants receiving Aripiprazole depot $(n=57)$. Please note: Values represent frequency (percentage) unless otherwise stated.

\begin{tabular}{lc}
\hline Male / Female & $31(54.4) / 26(45.6)$ \\
Age (years) & $\mathrm{M}=40.4(\mathrm{SD}=14.7 ;$ range $=20-87)$ \\
Ethnicity & $22(38.6)$ \\
White & $19(33.3)$ \\
Black & $5(8.8)$ \\
Asian & $11(19.3)$ \\
Mixed/Other & \\
Employment status & $44(77.2)$ \\
Unemployed & $7(12.3)$ \\
Employed & $4(7.0)$ \\
Student & $2(3.5)$ \\
Retired & \\
Accommodation status & $40(70.2)$ \\
Independent & $11(19.3)$ \\
Supported & $3(5.3)$ \\
Living with parents & $3(5.3)$ \\
Other/Unknown & \\
Diagnosis/Diagnoses & $42(73.7)$ \\
Schizophrenia & $6(10.5)$ \\
Schizoaffective disorder & $5(8.8)$ \\
Delusional disorder & $4(7.0)$ \\
Bipolar disorder & $2(3.5)$ \\
Emotionally unstable personality disorder & $5(8.8)$ \\
Substance abuse disorder &
\end{tabular}


Health behaviours

Smoking

Safety and effectiveness of Aripiprazole Depot

Alcohol abuse

$26(45.6)$

Illicit drug use

$19(33.3)$

Medical History

Obesity

Diabetes

Hypertension

Other

Previous medication (prior to Aripiprazole)

Olanzapine

$23(40.4)$

Risperidone

$20(35.1)$

Paliperidone

$6(10.5)$

Quetiapine

$9(15.8)$

Pipotiazine

Amisulpride

Flupentixol

Zuclopenthixol

Other

None

$\mathrm{M}=$ mean number/score; $\mathrm{SD}=$ standard deviation; Two patients' accommodation status was unknown and one patient had no fixed abode. Six patients had (different) comorbid diagnoses ( 2 schizophrenia + delusional disorder; 1 schizophrenia + bipolar; 1 schizophrenia + substance abuse disorder; 1

schizophrenia + schizoaffective + substance abuse disorder; 1 bipolar + substance abuse disorder $)$ - as such, percentages in each row do not add up to $100 \%$; Medical history 'Other' included epilepsy, mild mental retardation, Asperger's syndrome, HIV+, hypercholesteremia, hyperlipidemia, hypothyroidism, Fowler's syndrome, Sickle cell disease, and priapism; Eighteen patients were receiving more than one antipsychotic medication (10 receiving two medications, 7 receiving three medications, 1 receiving four medications) - as such, percentages in each row do not add up to $100 \%$; 'Other' previous medications included sulpiride and haloperidol.

Table 2. Aripiprazole PO and Depot treatment. Please note: Values

represent frequency (percentage) unless otherwise stated.

Aripiprazole $\mathrm{PO}$ administration $(n=54)$

Duration (median months (range))

$9.7(0.4-117.0)$

Maximum dosage (mg)

10

$13(24.1)$

15

$31(57.4)$

$8(14.8)$

30

$2(3.7)$

Reason for switch (to Aripiprazole depot)

Side effects

Non-Compliance

Side effects + Non-Compliance

Side effects + Not effective

Non-Compliance + Not effective

Non-Compliance + Patient request

Aripiprazole Depot administration $(n=57)$

Number stopped at time of study

$\underline{\text { Reason for stopping }(n=15)}$

Ineffective

Side effects

$4(26.7)$ 
Safety and effectiveness of Aripiprazole Depot

Non-Compliance

Patient request / Unknown

Duration (median months (range))

Patients who stopped Depot $(n=15)$

Patients with ongoing Depot $(n=42)$
$1(6.7)$

$4(26.7)$

$4.0(0.4-20.7)$

$14.5(3.0-41.0)$ 


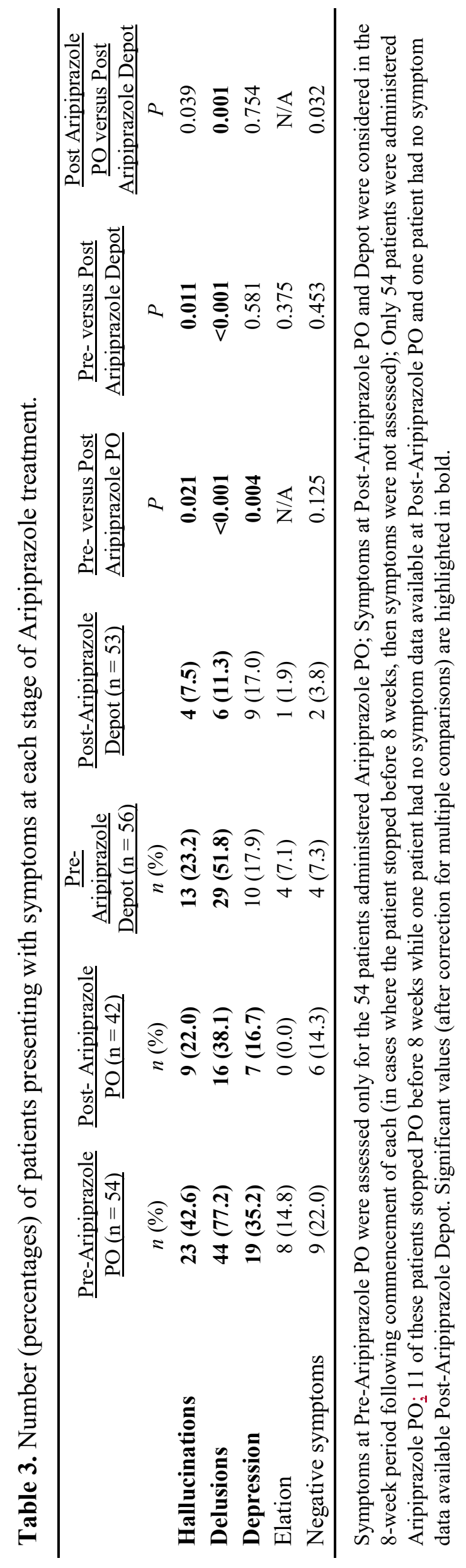

음 


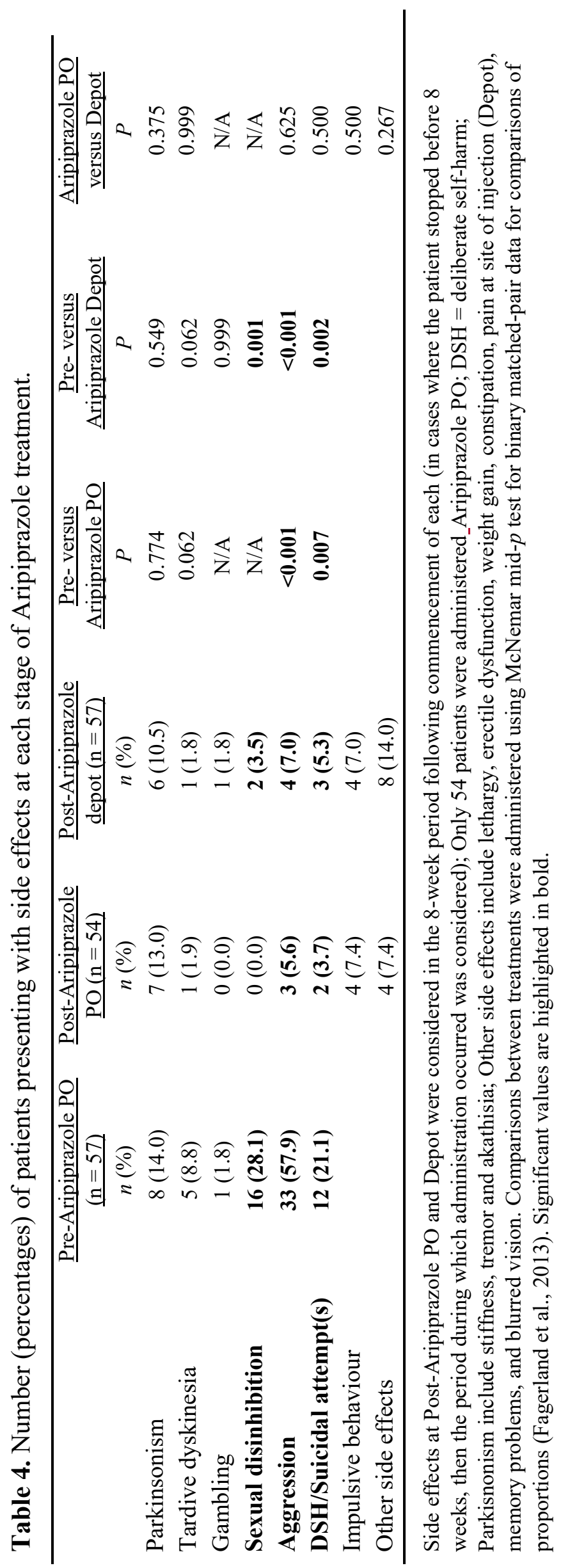

ำ 